\title{
Prevalence, genetic diversity and implications for public health of Enterocytozoon bieneusi in various rodents from Hainan Province, China
}

\author{
Wei Zhao ${ }^{1}$, Huanhuan Zhou ${ }^{2}$, Ling Yang ${ }^{1}$, Tianming $\mathrm{Ma}^{2}$, Jingguo Zhou ${ }^{2}$, Haiju Liu ${ }^{2}$, \\ Gang $\mathrm{Lu}^{2}$, and Huicong Huang ${ }^{1}$ \\ ${ }^{1}$ Wenzhou Medical University \\ ${ }^{2}$ Hainan Medical University
}

June 11, 2020

\begin{abstract}
Rodents, globally overpopulated, are important source for zoonotic disease transmission to humans, including Enterocytozoon bieneusi (one of the most prevalent zoonotic pathogen). Here, we studied the prevalence and performed genetic analysis of E. bieneusi in rodents from the Hainan province of China by the amplification of the internal transcribed spacer (ITS) region of rDNA of E. bieneusi using PCR. Six hundred and three fresh fecal samples were gathered from 369 wild rats, 117 bamboo rats, 93 Asiatic brush-tailed porcupine and 24 red-bellied squirrels. The average rate of infection of E. bieneusi was $15.8 \%$ $(95 / 603)$ with $18.7 \%(69 / 369)$ in wild rats, $11.9 \%(25 / 210)$ in farmed rodents and $4.2 \%(1 / 24)$ for red-bellied squirrels. Sixteen E. bieneusi genotypes were identified, including nine known genotypes (D, Type IV, PigEBITS7, Peru8, Peru11, ESH02, S7, EbpA and CHG5), and seven novel genotypes (HNR-I to HNR-VII). Genotype D $(44.2 \%, 42 / 95)$ predominated, followed by PigEBITS7 (20.0\%, 19/95), HNR-VII (15.8\%, 15/95), Type IV (5.3\%, 5/95), HNR-III (2.1\%, 2/95), HNR-VI (2.1\%, 2/95) and each of the remaining 10 genotypes $(1.1 \%, 1 / 95)$. This is the first report on the identification of E. bieneusi in rodents from Hainan, China. The zoonotic potential of the identified E. bieneusi genotypes suggested that the rodents posed a serious threat to the local inhabitants. Thus, measures need to be taken to control the population of wild rats in the areas investigated in this study, along with identification of safe methods of disposal of farmed rodent feces. Additionally, the local people should be made aware of the risk of disease transmission from rodents to humans.
\end{abstract}

Prevalence, genetic diversity and implications for public health of Enterocytozoon bieneusi in various rodents from Hainan Province, China

Running title: Enterocytozoon bieneusi in rodents

Wei Zhao ${ }^{1,2+}$, Huanhuan Zhou ${ }^{2+}$, Ling Yang ${ }^{1}$, Tianming $\mathrm{Ma}^{2}$, Jingguo Zhou ${ }^{2}$, Haiju Liu ${ }^{2}$, Gang Lu$^{2 *}$, Huicong Huang ${ }^{*}$

\section{Emails}

Wei Zhao: hayidazhaowei@163.com

Huanhuan Zhou: zhh931218@163.com

Ling Yang:82504366@qq.com

Tianming Ma: 619970219@qq,com

Jingguo Zhou: 842183423@qq.com 
Haiju Liu: 1512470133@qq.com

Gang Lu: luganghn@163.com

Huicong Huang: hhc@wmu.edu.cn

\section{Affiliations:}

${ }^{1}$ Department of Parasitology, Wenzhou Medical University, Wenzhou, Zhejiang Province, 325035, China.

${ }^{2}$ Department of Pathogenic Biology, Hainan Medical University, Haikou, Hainan, China; Hainan Medical University-The University of Hong Kong Joint Laboratory of Tropical Infectious Diseases, Hainan Medical University, Haikou, Hainan, China; Key Laboratory of Tropical Translational Medicine of Ministry of Education, Hainan Medical University, Haikou 571199, China

+ These authors have contributed equally to this work.

*Corresponding author: Huicong Huang: hhc@wmu.edu.cn; Gang Lu:

luganghn@163.com

\section{SUMMARY}

Rodents, globally overpopulated, are important source for zoonotic disease transmission to humans, including Enterocytozoon bieneusi (one of the most prevalent zoonotic pathogen). Here, we studied the prevalence and performed genetic analysis of E. bieneusi in rodents from the Hainan province of China. Six hundred and three fresh fecal samples were gathered from 369 wild rats, 117 bamboo rats, 93 Asiatic brush-tailed porcupine and 24 red-bellied squirrels. Species of the wild rats identification was done by the amplification of a 421 bp region of the cytb gene in the fecal DNA using PCR. Genotype analysis was done by the amplification of the internal transcribed spacer (ITS) region of rDNA of E. bieneusi using PCR. Seven wild rat species, including Asian house rats (Rattus tanezumi) $(\mathrm{n}=134)$, brown rats (Rattus norveqicus $)(\mathrm{n}=56)$, Chinese white-bellied rats (Niviventer confucianus ) $(\mathrm{n}=51)$, Edward's long tailed rats (Leopoldamys edwardsi ) ( $\mathrm{n}$ $=38)$, Indo-Chinese forest rats (Rattus andamanensis $)(\mathrm{n}=54)$, lesser rice-field rat (Rattus losea $)(\mathrm{n}=44)$ and muridae (Niviventer fulvescens $)(\mathrm{n}=10)$ were identified. The average rate of infection of $E$. bieneusi was $15.8 \%(95 / 603)$ with $18.7 \%(69 / 369)$ in wild rats, $11.9 \%(25 / 210)$ in farmed rodents and $4.2 \%(1 / 24)$ for red-bellied squirrels. Sixteen $E$. bieneusi genotypes were identified, including nine known genotypes (D, Type IV, PigEBITS7, Peru8, Peru11, ESH02, S7, EbpA and CHG5), and seven novel genotypes (HNR-I to HNRVII). Genotype D (44.2\%, 42/95) predominated, followed by PigEBITS7 (20.0\%, 19/95), HNR-VII (15.8\%, 15/95), Type IV $(5.3 \%, 5 / 95)$, HNR-III $(2.1 \%, 2 / 95)$, HNR-VI $(2.1 \%, 2 / 95)$ and each of the remaining 10 genotypes $(1.1 \%, 1 / 95)$. This is the first report on the identification of $E$. bieneusi in rodents from Hainan, China. The zoonotic potential of the identified E. bieneusi genotypes suggested that the rodents posed a serious threat to the local inhabitants. Thus, measures need to be taken to control the population of wild rats in the areas investigated in this study, along with identification of safe methods of disposal of farmed rodent feces. Additionally, the local people should be made aware of the risk of disease transmission from rodents to humans.

KEYWORDS : Enterocytozoon bieneusi, rodent, genotype, ITS, zoonotic

\section{INTRODUCTION}

Enterocytozoon bieneusi is a prevalent pathogen in humans and an important zoonotic agent in animals worldwide (Fayer and Santin-Duran, 2014). The most common clinical symptom of E. bieneusi infections is diarrhea for patients with Acquired Immunodeficiency Syndrome (AIDS) or other immunocompromising conditions while asymptomatic infections are common for healthy persons (Matos et al., 2012). Mostly, human acquire infections by ingesting the infectious spores of E. bieneusiwhich potential sources include: water, soil, environmental surfaces, fecal contamination, and improper manure or irrigation water practices in growing fruits and vegetables (Zhao et al., 2014; Li et al., 2012; Li et al., 2019; Santin and Fayer 2011). 
Thus, understanding the source and mechanism of transmission of E. bieneusi could prevent its spread in humans.

Previous studies detected E. bieneusi using PCR-based assays with appropriate gene markers (Santin and Fayer 2009). More than 500 genotypes, of which 142 infected humans and 49 infected both humans and animals, were identified through the internal transcribed spacer (ITS) sequence analysis of rDNA of the E. bieneusi (Zhou et al., 2020; Li et al., 2019; Zhao et al., 2020). These genotypes can be grouped into 11 genetically isolated clusters by phylogenetic analysis (Li et al., 2019). Group 1 is the largest group containing more than 300 genotypes, including $87.6 \%$ (132/142) human-pathogenic genotypes (Gong et al., 2019; Li et al., 2019). Meanwhile, 85.7\% (42/49) zoonotic genotypes also belong to this group (Zhou et al., 2020; Li et al., 2019). In Group 2, up to 100 genotypes are considered to be adapted to ruminants; however, contrary to previous reports, a broader host range was indicated for some genotypes, such as genotypes I, J, BEB6, BEB4 and CHN3 are also commonly found in humans, enhancing their importance for public health (Li et al., 2019). Genotypes in Groups 3 to 11 have been mainly reported in specific hosts or wastewater (Guo et al., 2014). Group 1 or 2 are frequently reported in pets, non-human primates, wildlife, and livestock (pigs, cattle, sheep, etc.) (Li et al., 2019; Zhao et al., 2020). However, the mechanism of transmission of infection from a specific animal to humans is unclear.

Rodents have a high rate of multiplication as well as survival rate, which have resulted in their overpopulation. They are also the reservoirs or carriers of several types of zoonotic pathogens, includingE. bieneusi . Until now, sixty genotypes of E. bieneusi , including eighteen zoonotic genotypes (C, CZ3, D, BEB6, EbpC, Nig7, Peru6, Type IV, EbpA, PigITS7, H, S7, Peru8, S6, Peru16, J, Peru11, and PigITS5) have been identified in rodents, confirming their role in disease transmission (Li et al., 2020; Gui et al., 2019; Deng et al., 2016, 2018; Qi et al., 2015; Wang et al., 2019; Yu et al., 2019; Zhao et al., 2018; Perec-Matysiak et al., 2015; Danišová et al., 2015; Guo et al., 2014; Roelling et al., 2015; Sak et al., 2011; Cama et al., 2007)(Table 1) .

Previous studies have reported E. bieneusi infections in eight rodent species, including, brown rats (Rattus norvegicus), bamboo rats (Rhizomys sinensis), house mice (Mus musculus), Bower's white-toothed rats (Berylmys bowersi), Edward's long-tailed rats (Leopoldamys edwardsi), Chipmunks (Eutamias asiaticus ), Chinchillas (Chinchilla lanigera), and red-bellied squirrels (Callosciueus erythraeus ) in China. These studies involved five provinces with infection rates ranging from 3.6\% to 35.1\% (Gui et al., 2019; Deng et al., 2016, 2018; Qi et al., 2015; Wang et al., 2019; Yu et al., 2019; Zhao et al., 2018). Hainan Province of China where is rich in rodents and they closely contact with humans and other animals. Meanwhile, there is high prevalence of E. bieneusi in farmed, household, and wild animals (Zhao et al., 2020; Zhou et al., 2019; Chen et al., 2019; Zhou et al., 2020), and was identified in humans with diarrhea (unpublished data) in Hainan, China. Thus, it is necessary to understand $E$. bieneusi epidemiology in rodents to prevent pathogen infection in humans as well as in other animals. This study explored the prevalence of E. bieneusi in rodents in several areas of Hainan Province of China, to evaluate the zoonotic potential of the isolates at the genotype level.

\section{MATERIALS AND METHODS}

\subsection{Ethical statement}

The research protocol was reviewed and approved by the Research Ethics Committee and the Animal Ethical Committee of Hainan Medical University. All wild rats were captured with traps and killed by $\mathrm{CO}_{2}$ inhalation. Fecal samples were collected from other rodent farms with the consent of farm owners. During the experimental process, all animals were handled and cared for according to the Chinese Laboratory Animal Administration Act of 1998.

\subsection{Rodent fecal sample collection}

Six hundred and three fecal specimens were collected from 210 farmed rats, 369 wild rats, and 24 red-bellied squirrels from Hainan, China between December 1, 2017 and October 31, 2019 (Table 2 ). For capturing the wild rats, 20 cage traps were installed at each location containing peanut/sesame butter and sunflower seeds as the bait. The cages were positioned at sunset 5 meters apart in transects and collected before sunrise. 
Within $48 \mathrm{~h}$ of capture, the wild rats were euthanized through $\mathrm{CO}_{2}$ inhalation, followed by the collection of fresh fecal specimens ( $\sim 500 \mathrm{mg})$ from the intestines.

For the farmed rats, a total of 117 bamboo rats and 93 Asiatic brush-tailed porcupine feces samples were procured from tanks each housing 1 to 3 animals. A single fecal specimen $(\sim 15 \mathrm{~g})$ was gathered from each tank (using disposable gloves), and approximately $30 \%$ of the total animals of each farms were collected, to minimize duplicate sampling.

Additionally, 24 fecal samples were collected from red-bellied squirrels which were captured from the Jianfeng Ling (18²3'-18deg52' N, 108deg44'-109deg02' E).

\subsection{DNA extraction}

Each fecal specimen was centrifuged at 1,500xg for $10 \mathrm{~min}$ at room temperature, followed by isolation of genomic DNA from each sample ( $\sim 200 \mathrm{mg}$ ) using QIAamp DNA Mini Stool Kit (Qiagen, Germany) following manufacturer's instructions. The lysis temperature was raised to $95 \mathrm{deg} \mathrm{C}$ to obtain higher yield. AE elution buffer $(200 \mathrm{~mL})$ was used to elute the DNA, followed by storage at -20degC for further PCR analysis.

\subsection{Identification of wild rat species}

A 421 bp sequence of the cytochrome b (cytb) gene in the fecal DNA was amplified using PCR to identify the rat species following a previously identified method (Verma and Singh, 2003). The PCR cycle had the following parameters: $94 \mathrm{deg} \mathrm{C}$ for $5 \mathrm{~min}, 35$ cycles at $94 \mathrm{deg} \mathrm{C}$ for $30 \mathrm{~s}$; $51 \mathrm{degC}$ for $30 \mathrm{~s} ; 72 \mathrm{degC}$ for $30 \mathrm{~s}$, and finally $72 \mathrm{degC}$ for $5 \mathrm{~min}$.

\subsection{Genotyping of E. bieneusi}

Nested PCR amplification of the ITS region was done to identify and genotype E. bieneusi using TaKaRa TaqDNA Polymerase along with genotype BEB6 DNA from deer (positive control) and $2 \mu \mathrm{L}$ distilled water (negative control). Buckholt and colleagues designed the primers and cycle parameters (Buckholt et al., 2002). The PCR products were analyzed using 1.5\% agarose gel electrophoresis, followed by visualization by DNAGREEN staining (Tiandz, Inc., China).

\subsection{DNA sequencing and analysis}

The PCR products that were E. bieneusi positive underwent bidirectional sequencing (Sangon Biotech Co., Ltd., China). Further PCR products were sequenced as necessary. Basic Local Alignment Search Tool (BLAST) and ClustalX 1.83 were used for genotyping of the E. bieneusi isolates by comparing the identified nucleotide sequence with the published GenBank sequences. The genotypes were labeled following established nomenclature based on the $243 \mathrm{bp}$ of the ITS region of E. bieneusi .

\subsection{Phylogenetic analysis}

A neighboring-joining phylogenetic tree was constructed by the Mega $\mathrm{X}$ software using the Kimura-2parameter model and with 1,000 replicates to evaluate the relationship between the genotypes identified in this study and to confirm the genegroup.

\subsection{Nucleotide sequence accession numbers}

The GenBank database accession number of the identified nucleotide sequences were MN267052 to MN267057 and MN931659.

\section{RESULTS}

\section{Identification of the rat species}

PCR gene sequencing showed that the 369 samples of wild rats contained 38 Edward's long tailed rats (Leopoldamys edwardsi), 44 lesser rice-field rat (Rattus losea), 134 Asian house rats (Rattus tanezumi ), 10 muridae (Niviventer fulvescens ), 56 brown rats (Rattus norveqicus ), 54 Indo-Chinese forest rats (Rattus andamanensis) and 33 Chinese white-bellied rats (Niviventer confucianus). All cytb sequences 
shared $99-100 \%$ similarity to the following reference sequences: MG748345 for $R$. tanezumi, MG748255 for $N$. fulvescens, KT808632 for $R$. norveqicus, MG748260 for $R$. andamanensis, MG748257 for R. losea, KP992477 for L. edwardsi and JF714942 for $N$. confucianus.

\section{Prevalence of E. bieneusi}

We detected E. bieneusi in $15.8 \%$ (95/603) of the rodent samples, including $18.7 \%$ (69/369) of the wild rats, $4.2 \%(1 / 24)$ of the red-bellied squirrels, and $11.9 \%(25 / 210)$ of the farmed rodents (Table 2). Among the wild rats, lesser rice-field rats have the highest prevalence rate of E. bieneusi $(16 / 44,36.4 \%)$, followed by Asian house rats $(31 / 134,23.1 \%)$, Chinese white-bellied rats $(6 / 33,18.2 \%)$, brown rats $(8 / 56,14.3 \%)$, Indo-Chinese forest rats $(5 / 54,9.3 \%)$ and Edward's long-tailed rats $(3 / 38,7.9 \%)$. None of the 10 Muridae species were infected with E. bieneusi . Among the farmed rodents, the rate of prevalence of E. bieneusi in bamboo rats $(18 / 117,15.4 \%)$ was higher than that of the Asiatic brush-tailed porcupines $(7 / 93,7.5 \%)$. Only one of the 24 (4.2\%) red-bellied squirrel was infected with E. bieneusi .

\section{Characterization and distribution of the genotypes of $E$. bieneusi}

We identified 16 genotypes containing 41 polymorphic sites, including nine known genotypes (D, Type IV, PigEBITS7, Peru8, Peru11, ESH02, S7, EbpA and CHG5) and seven novel genotypes (HNR-I to HNRVII (MN267052 to MN267057 and MN267057)) based on the ITS sequencing of the 95 E. bieneusi isolates (data not show). Amongst them, genotype D (44.2\%, 42/95) predominated, followed by PigEBITS7 (20.0\%, 19/95), HNR-VII (15.8\%, 15/95), Type IV (5.3\%, 5/95), HNR-III $(2.1 \%, 2 / 95)$, HNR-VI $(2.1 \%, 2 / 95)$ and each of the remaining 10 genotypes $(1.1 \%, 1 / 95)$ (Table 2) .

Nucleotide sequence analysis showed that the novel genotypes HNR-I, HNR-II, HNR-VI, and HNR-VII had the largest similarity with genotypes Peru8 (MF476880), Type IV (KP994661) SCR06 (MK909573) and S (AY945809) with one base insert at position 10 (a single nucleotide "A" insertion), 244 (G-A), 222 (C-A) and 134 (T-C), respectively. Genotype HNRM-III had two base differences at positions 118 (G-T) and 144 (A-G) compared to genotype HLJ-I (KJ475402) from pigs in Heilonjiang, China. In contrast, genotypes HNR-IV and HNR-V had the largest similarity with genotypes YNM1 (MG999511) and D (KU557672), with six and five base differences at positions 3 (A-G), 65 (G-T), 226 (G-A), 234 (G-A), 235 (G-T) and 243 (G-T), and 226 (G-A), 234 (G-A), 235 (G-T), 237 (G-A) and 243 (G-T), respectively.

We observed a varied distribution pattern of the E. bieneusigenotypes among different rodent species (Table 2) . Genotype D was found in all rodent species which were positive for the pathogen. Genotypes PigEbITS7, Type IV, Peru 8, EbpA, ESH-02, HNR-I to HNR-III and HNR-VII were found in wild rodents with genotype PigEbITS7 and Type IV in Asian house rats, Chinese white-bellied rats and brown rats; ESH-02 in Asian house rats; Peru 8, HNR-I and HNR-II in brown rats; HNR-III in Edward's long-tailed rats and Indo-Chinese forest rats, genotype HNR-VII in lesser rice-field rats; EbpA in Asian house rat. On the contrary, genotypes S7, CHG5, Peru 11 and HNR-IV to HNR-VI were present in farmed rodents with genotypes HNR-VI, S7, and CHG5 in Asiatic brush-tailed porcupines and genotypes Peru 11, HNR-IV and HNR-V in bamboo rats(Table 2). Genotype D was detected in the E. bieneusiisolate from the red-bellied squirrel.

\section{Phylogenetic analysis}

The phylogenetic analysis of the ITS region of E. bieneusidivided the identified genotypes into the following four groups: Group $1(\mathrm{n}=13)$, Group $2(\mathrm{n}=1)$, Group 12, and the novel Group $13(\mathrm{n}=1)$ (Figure 1) .

\section{DISCUSSION}

This study is the first report on the identification of E. bieneusi in rodents in Hainan, the southernmost province of China. Currently, there are 14 studies reporting the presence of E. bieneusi in rodents from six different countries. These studies describe the rate of prevalence of E. bieneusi infection among the rodents in the range of 1.1-100.0\% ( $\mathrm{Li}$ et al., 2020; Gui et al., 2019; Deng et al., 2016, 2018; Qi et al., 2015; Wang et al., 2019; Yu et al., 2019; Zhao et al., 2018; Perec-Matysiak et al., 2015; Daniaová et al., 2015; Guo et al., 2014; Roelling et al., 2015; Sak et al., 2011; Cama et al., 2007) (Table 1 ). There were geographical 
location-based variation in the average rate of prevalence of E. bieneusi in rodents: $87.55 \%(7 / 8)$ in Peru (Cama et al., 2007), 38.9\% (121/311) in Poland (Perec-Matysiak et al., 2015), 35.9\% (52/145) in the United States (Guo et al., 2014; Roelling et al., 2015), 10.7\% (31/289) at the Switzerland and Germany border (Sak et al., 2011), 12.2\% (278/2272) in China (Li et al., 2020; Gui et al., 2019; Deng et al., 2016, 2018, 2020; Qi et al., 2015; Wang et al., 2019; Yu et al., 2019; Zhao et al., 2018), and 1.1\% (3/280) in Slovakia (Danišová et al., 2015). These studies also reported species-based variation in the prevalence rate of $E$. bieneusi infection: $5.1 \%$ for bamboo rats, $87.5 \%$ for guinea-pigs, $48.3 \%$ for prairie dogs, $39.1 \%$ for voles, $24.3 \%$ for hamsters, $16.7-42.9 \%$ for squirrels, $4.0-7.9 \%$ for rats, $3.6-71.4 \%$ for chipmunks, and $1.1-87.5 \%$ for mice (Table 1) . Notably, except for China and the United States, only one study was performed in each of the other countries and thus further large-scale surveillance studies should be conducted to ascertain these findings. We also found a variation in the prevalence rate of $E$. bieneusiinfection in our rodents. Thus, the rate of prevalence of E. bieneusi was $16.6 \%$ in the wild rats, $4.2 \%$ in the red-bellied squirrels, and $11.9 \%$ in the farmed rodents. Wild rats showed a significantly higher rate of E. bieneusi infection compared with farmed rats and squirrels.

Among the 16 identified E. bieneusi genotypes, genotypes D, Peru8, PigEbITS7, Type IV, Peru11, and EbpA are known human pathogens ( $\mathrm{Li}$ et al., 2019). Genotype D was the predominant genotype which was found in $44.2 \%(42 / 95)$ of E. bieneusi isolates. This genotype was widely distributed and present in all sampled rodent species. It is also commonly found in human infections in [?]20 countries and has been isolated from [?]25 animal species and water samples (Li et al., 2019). Genotype PigEbITS7 in Asian house rats, brown rats, and Chinese white-bellied rats. This genotype was originally identified in pigs in Massachusetts, USA, and in immunocompromised patients in Ahvaz in Iran, and Gangxi and Henan in China (Buckholt et al., 2002; Liu et al.,2017; Wang et al., 2013). In addition to pigs and humans, genotype PigEbITS7 has been identified in monkeys and bamboo rats from China (Wei et al., 2019; Zhao et al., 2020). Genotypes Peru8, type IV, and Peru11 were detected in a single rat species but are known to be human and animal pathogens (Li et al., 2019). Thus, the identification of the above mentioned six genotypes in rodents indicated the transmission of parasites from pathogen-infected rodents to humans as well as other animals.

The remaining two known genotypes ESH-02 and S7 were found in Asian house rats and Asiatic brushtailed porcupines, respectively. Genotype ESH-02 (also named Ind 1) was originally identified in wastewater treatment plant effluents in Shanghai, China (Ma et al., 2016), and also in renal transplant recipients and AIDS patients in India (Khanduja et al., 2017). There are no published reports of the presence of this genotype in any animal species. This study confirmed for the first time that genotype ESH-02 can infect rats, suggesting its zoonotic potential. Genotype S7 (also named CHY1) was previously identified in an immunosuppressed patient in the Netherlands in 2009 (ten Hove et al., 2009), yark in Henan, China (Li et al., 2015), chipmunks and rabbits from Sichuan, China (Deng et al., 2018) and experimental rats in Henan, China (Li et al., 2020). We found that this genotype of E. bieneusi was also found in Asiatic brush-tailed porcupines. These finding indicated that genotype S7 has a wide range of animal reservoirs and potential for zoonotic transmission. Further studies should be conducted to explore additional animal reservoirs of these genotypes.

In this study, 13/16 (81.3\%) genotypes and 95.8\% (91/95) of theE. bieneusi isolates belonged to Group 1. The genotypes in this group has been identified in several hosts, such as humans, and possess a high potential for cross-species and zoonotic transmission of E. bieneusi (Li et al., 2019). Group 1 was deemed zoonotic based on the prevalence of genotypes such as Type IV, D, Peru11, EbpC, and Peru8 in several animal hosts (Li et al., 2019). The fact above suggesting that the E. bieneusi -infected rodents posed a serious threat to the local inhabitants. Meanwhile, the identification of genotype HNR-VII belonging to the novel Group 13, was a unique epidemiological feature of E. bieneusi in rodents in Hainan Province of China.

\section{CONCLUSIONS}

Our novel data demonstrated a high rate of prevalence of $E$. bieneusi infection in various rodent species in Hainan, China. The finding of zoonotic E. bieneusi genotypes (PigEbITS7, Peru8, D, Type IV, Peru11, EbpA, S7, and ESH-02) in rodents suggested they may pose serious public health threats in the area. 
Moreover, the seven novel genotypes provided novel insights into the genotypic variations of $E$. bieneusi. Adequate control of rodents and public education on the management of rodent feces should be implemented in these areas.

\section{AUTHOR CONTRIBUTIONS}

WZ, H-C H and GL conceived the study and contributed to the design. H-H Z, T-M M, J-G Z and H-J D contributed to acquisition of samples. H-H Z, J-G Z, H-J D and T-M M performed experiments. WZ and $\mathrm{H}-\mathrm{H} \mathrm{Z}$ contributed to data analysis. WZ contributed to writing the manuscript. H-C H and GL review and editing the manuscript. GL and WZ obtained funding. H-C H provided technical support and constructive discussion. All authors approved the final version to be published and agreed to be accountable for all aspects of the manuscript.

\section{COMPETING INTERESTS}

The authors declared no competing interests.

\section{ACKNOWLEDGMENTS}

This work was supported partially by the Innovation Research Team Project of Hainan Natural Science Foundation (2018CXTD340) and the National Natural Science Foundation of China (No. 81672072 and No. 81760378). The sponsors played no roles in the study design, or in the collection, analysis, or interpretation of the data, in writing the report, or in the decision to submit the article for publication.

\section{DATA AVAILABILITY STATEMENT}

The data that supports the findings of this study are available within the main body of the manuscript.

\section{FIGURE CAPTIONS}

Phylogenetic relationship of E. bieneusi genotypes identified here and other known genotypes deposited in GenBank was inferred by a neighboring-joining phylogenetic analysis of ITS sequences using the Kimura-2parameter model and with 1,000 replicates. Each sequence is identified by its accession number, host origin, and genotype designation. The E. bieneusi genotype CSK2 (KY706128) from white kangaroo was used as the outgroup. The black circles and squares indicate known and novel genotypes identified in this study, respectively.

\section{REFERENCES}

Buckholt, M. A., Lee, J. H., \& Tzipori, S. (2002). Prevalence ofEnterocytozoon bieneusi in swine: an 18-month survey at a slaughterhouse in Massachusetts. Applied and environmental microbiology , 68(5), $2595-2599$.

https://doi.org/10.1128/aem.68.5.2595-2599.2002.

Cama, V. A., Pearson, J., Cabrera, L., Pacheco, L., Gilman, R., Meyer, S., Ortega, Y., \& Xiao, L. (2007). Transmission of Enterocytozoon bieneusi between a child and guinea pigs. Journal of clinical microbiology, 45(8), 2708-2710.

https://doi.org/10.1128/JCM.00725-07.

Chen, L., Zhao, J., Li, N., Guo, Y., Feng, Y., Feng, Y., et al. (2019). Genotypes and public health potential of Enterocytozoon bieneusiand Giardia duodenalis in crab-eating macaques. Parasit Vectors . 12, 254. doi: 10.1186/s13071-019-3511-y.

Danišová, O., Valenčáková, A., Stanko, M., Luptáková, L., \& Hasajová, A. (2015). First report of Enterocytozoon bieneusi andEncephalitozoon intestinalis infection of wild mice in Slovakia.Annals of agricultural and environmental medicine : AAEM , 22(2), 251-252. https://doi.org/10.5604/12321966.1152075. 
Deng, L., Chai, Y., Luo, R., Yang, L., Yao, J., Zhong, Z., Wang, W., Xiang, L., Fu, H., Liu, H., Zhou, Z., Yue, C., Chen, W., \& Peng, G. (2020). Occurrence and genetic characteristics of Cryptosporidiumspp. and Enterocytozoon bieneusi in pet red squirrels (Sciurus vulgaris ) in China. Scientific reports , 10(1), 1026. https://doi.org/10.1038/s41598-020-57896-w.

Deng, L., Li, W., Zhong, Z., Chai, Y., Yang, L., Zheng, H., Wang, W., Fu, H., He, M., Huang, X., Zuo, Z., Wang, Y., Cao, S., Liu, H., Ma, X., Wu, K., \& Peng, G. (2018). Molecular characterization and new genotypes of Enterocytozoon bieneusi in pet chipmunks (Eutamias asiaticus ) in Sichuan province, China. BMC microbiology , 18(1), 37. https://doi.org/10.1186/s12866-018-1175-y.

Fayer, R., and Santin-Duran, M. (2014). "Epidemiology of microsporidia in human infections," in Microsporidia: Pathogens of Opportunity, 1st Edn, eds L. M. Weiss and J. J. Becnel (Chichester: John Wiley E Sons, Inc .), 1-64. doi.10.1002/9781118395264

Gong, B., Yang, Y., Liu, X., Cao, J., Xu, M., Xu, N., Yang, F., Wu, F., Li, B., Liu, A., \& Shen, Y. (2019). First survey ofEnterocytozoon bieneusi and dominant genotype Peru6 among ethnic minority groups in southwestern China's Yunnan Province and assessment of risk factors. PLoS neglected tropical diseases , 13(5), e0007356. https://doi.org/10.1371/journal.pntd.0007356 .

Gui, B. Z., Zou, Y., Chen, Y. W., Li, F., Jin, Y. C., Liu, M. T., Yi, J. N., Zheng, W. B., \& Liu, G. H. (2020). Novel genotypes and multilocus genotypes of Enterocytozoon bieneusi in two wild rat species in China: potential for zoonotic transmission. Parasitology research , 119(1), 283-290.

https://doi.org/10.1007/s00436-019-06491-8.

Guo, Y., Alderisio, K. A., Yang, W., Cama, V., Feng, Y., \& Xiao, L. (2014). Host specificity and source of Enterocytozoon bieneusigenotypes in a drinking source watershed. Applied and environmental microbiology , 80(1), 218-225.https://doi.org/10.1128/AEM.02997-13 .

Khanduja, S., Ghoshal, U., Agarwal, V., Pant, P., \& Ghoshal, U. C. (2017). Identification and genotyping of Enterocytozoon bieneusiamong human immunodeficiency virus infected patients. Journal of infection and public health , 10(1), 31-40.

https://doi.org/10.1016/j.jiph.2016.01.005.

Li, J., Jiang, Y., Wang, W., Chao, L., Jia, Y., Yuan, Y., Wang, J., Qiu, J., \& Qi, M. (2020). Molecular identification and genotyping of Enterocytozoon bieneusi in experimental rats in China.Experimental parasitology , 210, 107850 .

https://doi.org/10.1016/j.exppara.2020.107850.

Li, J., Qi, M., Chang, Y., Wang, R., Li, T., Dong, H., \& Zhang, L. (2015). Molecular Characterization of Cryptosporidium spp.,Giardia duodenalis, and Enterocytozoon bieneusi in Captive Wildlife at Zhengzhou Zoo, China. The Journal of eukaryotic microbiology , 62(6), 833-839.

https://doi.org/10.1111/jeu.12269.

Li, J., Shi, K., Sun, F., Li, T., Wang, R., Zhang, S., Jian, F., Ning, C., \& Zhang, L. (2019). Identification of human pathogenicEnterocytozoon bieneusi, Cyclospora cayetanensis, and Cryptosporidium parvum on the surfaces of vegetables and fruits in Henan, China. International journal of food microbiology , 307, 108292. https://doi.org/10.1016/j.ijfoodmicro.2019.108292.

Li, N., Xiao, L., Wang, L., Zhao, S., Zhao, X., Duan, L., et al. (2012). Molecular surveillance of Cryptosporidium spp., Giardia duodenalis, and Enterocytozoon bieneusi by genotyping and subtyping parasites in wastewater. PLoS Negl Trop Dis . 6(9):e1809. doi: 10.1371/journal.pntd.0001809.

Li, W., Feng, Y., \& Santin, M. (2019). Host Specificity of Enterocytozoon bieneusi and Public Health Implications. Trends in parasitology, 35(6), 436-451.https://doi.org/10.1016/j.pt.2019.04.004 . 
Liu, H., Jiang, Z., Yuan, Z., Yin, J., Wang, Z., Yu, B., Zhou, D., Shen, Y., \& Cao, J. (2017). Infection by and genotype characteristics of Enterocytozoon bieneusi in HIV/AIDS patients from Guangxi Zhuang autonomous region, China. BMC infectious diseases, 17(1), 684.

https://doi.org/10.1186/s12879-017-2787-9.

Ma, J., Feng, Y., Hu, Y., Villegas, E. N., \& Xiao, L. (2016). Human infective potential of Cryptosporidium spp., Giardia duodenalis and Enterocytozoon bieneusi in urban wastewater treatment plant effluents. Journal of water and health , 14(3), 411-423. https://doi.org/10.2166/wh.2016.192.

Matos, O., Lobo, M. L., and Xiao, L. (2012). Epidemiology ofEnterocytozoon bieneusi infection in humans. J. Parasitol. Res . 2012:981424. doi: 10.1155/2012/981424.

Perec-Matysiak, A., Buńkowska-Gawlik, K., Kváč, M., Sak, B., Hildebrand, J., \& Leśniańska, K. (2015). Diversity of Enterocytozoon bieneusi genotypes among small rodents in southwestern Poland.Veterinary parasitology , 214(3-4), 242-246.

https://doi.org/10.1016/j.vetpar.2015.10.018.

Qi, M., Luo, N., Wang, H., Yu, F., Wang, R., Huang, J., \& Zhang, L. (2015). Zoonotic Cryptosporidium spp. and Enterocytozoon bieneusiin pet chinchillas (Chinchilla lanigera) in China.Parasitology international , 64(5), 339-341.

https://doi.org/10.1016/j.parint.2015.05.00\%.

Roellig, D. M., Salzer, J. S., Carroll, D. S., Ritter, J. M., Drew, C., Gallardo-Romero, N., Keckler, M. S., Langham, G., Hutson, C. L., Karem, K. L., Gillespie, T. R., Visvesvara, G. S., Metcalfe, M. G., Damon, I. K., \& Xiao, L. (2015). Identification of Giardia duodenalis andEnterocytozoon bieneusi in an epizoological investigation of a laboratory colony of prairie dogs, Cynomys ludovicianus . Veterinary parasitology , 210(1-2), 91-97.

https://doi.org/10.1016/j.vetpar.2015.03.022.

Sak, B., Kváč, M., Květoňová, D., Albrecht, T., \& Piálek, J. (2011). The first report on natural Enterocytozoon bieneusi andEncephalitozoon spp. infections in wild East-European House Mice (Mus musculus musculus ) and West-European House Mice (M. m. domesticus ) in a hybrid zone across the Czech Republic-Germany border. Veterinary parasitology , 178(3-4), 246-250.

https://doi.org/10.1016/j.vetpar.2010.12.044.

Santín, M., \& Fayer, R. (2009). Enterocytozoon bieneusi genotype nomenclature based on the internal transcribed spacer sequence: a consensus. The Journal of eukaryotic microbiology , 56(1), 3438.https://doi.org/10.1111/j.1550-7408.2008.00380.x .

Santín, M., and Fayer, R. (2011). Microsporidiosis: Enterocytozoon bieneusi in domesticated and wild animals. Res. Vet. Sci. 90, 363-371. doi: 10.1016/j.rvsc.2010.07.014.

ten Hove, R. J., Van Lieshout, L., Beadsworth, M. B., Perez, M. A., Spee, K., Claas, E. C., \& Verweij, J. J. (2009). Characterization of genotypes of Enterocytozoon bieneusi in immunosuppressed and immunocompetent patient groups. The Journal of eukaryotic microbiology, 56(4), 388-393.

https://doi.org/10.1111/j.1550-7408.2009.00393.x.

Verma, S.K., Singh, L. (2003). Novel universal primers establish identity of an enormous number of animal species for forensic application. Molecualr Ecology . 3, 28-31. doi: 10.1046/j.1471-8286.2003.00340.x.

Wang, H., Liu, Q., Jiang, X., Zhang, Y., Zhao, A., Cui, Z., Li, D., Qi, M., \& Zhang, L. (2019). Dominance of zoonotic genotype D ofEnterocytozoon bieneusi in bamboo rats (Rhizomys sinensis ). Infection, genetics and evolution : journal of molecular epidemiology and evolutionary genetics in infectious diseases, 73, 113-118. https://doi.org/10.1016/j.meegid.2019.04.025. 
Wang, L., Zhang, H., Zhao, X., Zhang, L., Zhang, G., Guo, M., Liu, L., Feng, Y., \& Xiao, L. (2013). Zoonotic Cryptosporidium species andEnterocytozoon bieneusi genotypes in HIV-positive patients on antiretroviral therapy. Journal of clinical microbiology , 51(2), 557-563. https://doi.org/10.1128/JCM.02758-12.

Yu, F., Qi, M., Zhao, Z., Lv, C., Wang, Y., Wang, R., \& Zhang, L. (2019). The Potential Role of Synanthropic Rodents and Flies in the Transmission of Enterocytozoon bieneusi on a Dairy Cattle farm in China. The Journal of eukaryotic microbiology , 66(3), 435-441.

https://doi.org/10.1111/jeu.1268\%.

Zhao, W., Wang, J., Ren, G., Yang, Z., Yang, F., Zhang, W., Xu, Y., Liu, A., \& Ling, H. (2018). Molecular characterizations ofCryptosporidium spp. and Enterocytozoon bieneusi in brown rats (Rattus norvegicus ) from Heilongjiang Province, China.Parasites \& vectors , 11(1), 313.

https://doi.org/10.1186/s13071-018-2892-7.

Zhao, W., Zhang, W., Yang, F., Cao, J., Liu, H., Yang, D., et al. (2014). High prevalence of Enterocytozoon bieneusi in asymptomatic pigs and assessment of zoonotic risk at the genotype level.Appl. Environ. Microbiol . 80, 3699-3707. doi: 10.1128/AEM.00807-14.

Zhao, W., Zhou, H., Jin, H., Sun, L., Li, P., Liu, M., Qiu, M., Xu, L., Li, F., Ma, T., Wang, S., Yin, F., Li, L., Cui, X., Chan, J. F., \& Lu, G. (2020). Genotyping of Enterocytozoon bieneusi among captive long-tailed macaques (Macaca fascicularis ) in Hainan Province: High genetic diversity and zoonotic potential. Acta tropica , 201, 105211. https://doi.org/10.1016/j.actatropica.2019.105211 .

Zhou, H. H., Zheng, X. L., Ma, T. M., Qi, M., Zhou, J. G., Liu, H. J., Lu, G., \& Zhao, W. (2020). Molecular detection of Enterocytozoon bieneusi in farm-raised pigs in Hainan Province, China: infection rates, genotype distributions, and zoonotic potential. Parasite, 27, 12. https://doi.org/10.1051/parasite/2020009 .

Zhou, H. H., Zheng, X. L., Ma, T. M., Qi, M., Cao, Z. X., Chao, Z., Wei, L. M., Liu, Q. W., Sun, R. P., Wang, F., Zhang, Y., Lu, G., \& Zhao, W. (2019). Genotype identification and phylogenetic analysis ofEnterocytozoon bieneusi in farmed black goats (Capra hircus) from China's Hainan Province. Parasite, 26,62 .

https://doi.org/10.1051/parasite/2019064.

TABLE 1 Prevalence and distribution of Enterocytozoon bieneusi genotypes in rodents in the different countries or areas

\begin{tabular}{|c|c|c|c|c|}
\hline Country & $\begin{array}{l}\text { Species of Rodents ( } \\
\text { Latin name) }\end{array}$ & $\begin{array}{l}\text { Positive no. / } \\
\text { examined no. (\%) }\end{array}$ & Genotype/s (n) & References \\
\hline \multirow[t]{3}{*}{ China } & $\begin{array}{l}\text { Bamboo rats } \\
\text { (Rhizomys } \\
\text { sinensis) }\end{array}$ & $22 / 435(5.1)$ & $\begin{array}{l}\text { D }(17), \text { EbpA } \\
(1), \text { J (1), } \\
\text { PigEBITS7 (1), } \\
\text { BR1 (1), BR2 (1) }\end{array}$ & Wang et al., 2019 \\
\hline & $\begin{array}{l}\text { Brown rats } \\
\text { (Rattus } \\
\text { norvegicus) }\end{array}$ & $19 / 242(7.9)$ & $\begin{array}{l}\text { D (17); Peru6 } \\
(2)\end{array}$ & Zhao et al., 2018 \\
\hline & $\begin{array}{l}\text { Bower's } \\
\text { white-toothed } \\
\text { rats (Berylmys } \\
\text { bowersi) }\end{array}$ & $37 / 117(31.6)$ & $\begin{array}{l}\text { D (14), K (8), } \\
\text { PigEBITS7 } \\
\text { (22), Peru8 (2), } \\
\text { CQR-1 (10), } \\
\text { CQR-2 (15), } \\
\text { CQR-3 (1), } \\
\text { GDR-1(2), } \\
\text { GDR-2 (1), } \\
\text { GDR3 (1) }\end{array}$ & Gui et al., 2019 \\
\hline
\end{tabular}


Edward's

long-tailed rats

(Leopoldamys

edwardsi)

Experimental

rats $\#$

Chinchillas(Chinchilla 5/140 (3.6)

lanigera)

Chipmunks

(Eutamias

asiaticus)

Red-Bellied Tree

$24 / 144(16.7)$

Squirrels

(Callosciurus

erythraeus)

Red squirrels

(Sciurus vulgaris)

Rodents\#\#

Czech Republic and Germany border

Peru

Poland

Slovakia

\author{
East-European \\ House Mice (Mus \\ m. musculus) \\ West-European \\ House Mice (Mus \\ m. domesticus) \\ Guineapigs
(Cavia porcellus) \\ Striped field \\ mouse (Apodemus \\ agrarius) \\ yellow-necked \\ mouse (Apode- \\ musflavicollis) \\ Bankvole \\ (Myodesglareolus) \\ 14/127 (11.0) \\ $17 / 162(10.5)$ \\ $7 / 8(87.5)$ \\ 79/184 (42.9) \\ $18 / 60(30.0)$ \\ 18/46 (39.1)
}

House mouse

(Musmusculus)

Mice (Mus

musculus

musculus)
$14 / 291(4.8)$

61/314 (19.4)

8/199 (4.0)

EbpA (7), EbpC

(3), CHY1 (2),

N (1); SHR1 (1)

BEB6 (3), D (2) Qi et al., 2015

SCC-1 (17), Deng et al., 2018

SCC-2 (9), D (6),

CHY1 (5),

SCC-3 (5), Nig 7

(4), CHG9 (2),

$\mathrm{SCC}-4$ (1)

D (18), EbpC

(3), SC02 (1), CE01 (1), CE02

(1)

D $(27)$, SCC-2 (18), SCC-3 (12), RS01 (2), RS02

(2)

CHG14 (3),

BEB6 (2), D (2),

CHG2 (1)

EpbA (2), D (6), $\quad$ Sak et al., 2011

PigEBITS5 (4),

C (1), $\mathbf{H}$ (1)

CZ3 (4),

PigEBITS5 (3),

D (4), Peru 8

(4), S6 (1), C (1)

Preu 16 (7)

D (6), gorilla 1

(1), WR5

(1),WR7 (1),

WR8 (2)

D (2),WR4 (1),

WR6 (6), WR1

(1), WR9 (1)

D (2), WR6 (2),

WR10 (2), WR2

(1)

WR3 (1)

unknown (3)
Cama et al., 2007

Li et al., 2020

Deng et al., 2016

Deng et al., 2020

Yu et al., 2019

Sak et al., 2011

Perec-Matysiak et al., 2015

Perec-Matysiak et al., 2015

Perec-Matysiak et al., 2015

Perec-Matysiak et al., 2015

Danišová et al., 2015 


\begin{tabular}{|c|c|c|c|c|}
\hline \multirow[t]{7}{*}{ USA } & $\begin{array}{l}\text { Eastern gray } \\
\text { squirrel (Sciurus } \\
\text { carolinensis) }\end{array}$ & $11 / 34(32.4)$ & $\begin{array}{l}\text { Type IV (3), } \\
\text { WL4 (5), WW6 } \\
(2), \\
\text { PtEbV+WL21 } \\
(1)\end{array}$ & Guo et al., 2014 \\
\hline & $\begin{array}{l}\text { Eastern chipmunk } \\
\text { (Tamias striatus) }\end{array}$ & $5 / 7(71.4)$ & $\begin{array}{l}\text { Type IV (1), } \\
\text { WL4 (3), WL23 } \\
(1)\end{array}$ & Guo et al., 2014 \\
\hline & $\begin{array}{l}\text { Woodchuck } \\
\text { (Marmota } \\
\text { monax) }\end{array}$ & $5 / 5(100)$ & $\begin{array}{l}\text { Type IV+WL20 } \\
(1), \text { WL4 (2), } \\
\text { WL22 (1), WW6 } \\
(1)\end{array}$ & Guo et al., 2014 \\
\hline & $\begin{array}{l}\text { Deer mouse } \\
\text { (Peromyscus sp.) }\end{array}$ & $13 / 55(23.6)$ & $\begin{array}{l}\text { WL4 (10), WL23 } \\
(2), \text { WL25 (1) }\end{array}$ & Guo et al., 2014 \\
\hline & $\begin{array}{l}\text { Boreal red-backed } \\
\text { vole (Myodes } \\
\text { gapperi) }\end{array}$ & $1 / 5(20.0)$ & WL20+WL21(1) & Guo et al., 2014 \\
\hline & $\begin{array}{l}\text { Meadow vole } \\
\text { (Microtus } \\
\text { pennsylvanicus) }\end{array}$ & $3 / 10(30.0)$ & $\begin{array}{l}\text { Peru11 (1), } \\
\text { Peru11+Type } \\
\text { IV (1), } \\
\text { WL21+unknown } \\
(1)\end{array}$ & Guo et al., 2014 \\
\hline & $\begin{array}{l}\text { Prairie dogs } \\
\text { (Cynomys } \\
\text { ludovicianus) }\end{array}$ & $14 / 29(48.3)$ & Row (14) & $\begin{array}{l}\text { Roellig et al., } \\
2015\end{array}$ \\
\hline
\end{tabular}

\#Experimental rats including 104 wistar rats, 87 sprague dawley rats and 100 spontaneously hypertensive rats

\# \#Rodents including 168 brown rats (Rattus norvegicus) and31 house mice (Mus musculus )

Genotypes detected in humans are shown in bold in the table

TABLE 2 Prevalence and distribution of E. bieneusigenotypes in wild and farmed rodents in the Hainan Province of China

\begin{tabular}{|c|c|c|c|}
\hline Rodent species & No. of specimens & $\begin{array}{l}\text { E. bieneusi } \\
\text { No. of positive (\%) }\end{array}$ & $\begin{array}{l}\text { E. bieneusi } \\
\text { Genotype(s) (no. of } \\
\text { specimens) }\end{array}$ \\
\hline Wild rats & Wild rats & Wild rats & Wild rats \\
\hline $\begin{array}{l}\text { Asian house rats (Rattus } \\
\text { tanezumi) }\end{array}$ & 134 & $31(23.1)$ & $\begin{array}{l}\text { PigEbITS7 (16); D (12); } \\
\text { ESH-02 (1); Type-IV (1); } \\
\text { EbpA (1) }\end{array}$ \\
\hline $\begin{array}{l}\text { Brown Rats (Rattus } \\
\text { norvegicus) }\end{array}$ & 56 & $8(14.3)$ & $\begin{array}{l}\text { D (3); PigEbITS7 (1); } \\
\text { Type IV (1); Peru } 8 \text { (1); } \\
\text { HNR-I (1); HNR-II (1) }\end{array}$ \\
\hline $\begin{array}{l}\text { Edward's long-tailed rats } \\
\text { (Leopoldamys edwardsi) }\end{array}$ & 38 & $3(7.9)$ & D (2); HNR-III (1) \\
\hline $\begin{array}{l}\text { Muridae (Niviventer } \\
\text { fulvescens) }\end{array}$ & 10 & 0 & / \\
\hline $\begin{array}{l}\text { Indo-Chinese forest rats } \\
\text { (Rattus andamanensis) }\end{array}$ & 54 & $5(9.3)$ & $\begin{array}{l}\text { D (3), Type-IV (1), } \\
\text { HNR-III (1) }\end{array}$ \\
\hline
\end{tabular}


Lesser rice-field rats

(Rattus losea)

Chinese white-bellied rats

(Niviventer confucianus)

Subtotal

369

Wild squirrels

Red-bellied squirrels

(Callosciueus erythraeus)

Farmed rodents

Asiatic brush-tailed

porcupine (Atherurus

macrourus)

Bamboo rat

(Rhizomyidae)

Subtotal

Wild squirrels

24

Farmed rodents

93

117

210

Total

603
$69(18.7)$

$18(15.4)$

$25(11.9)$

$16(36.4)$

$6(18.2)$

Wild squirrels

1 (4.2)

Farmed rodents

$7(7.5)$

$95(15.8)$
HNR-VII (15), D (1)

D (3), PigEBITS7 (2), Type-IV (1)

D (24), PigEbITS7 (19), HNR-VII (15), Type IV

(4), HNR-III (2), Peru 8

(1), EbpA (1), ESH-02

(1), HNR-I (1) and

HNR-II (1)

Wild squirrels

D (1)

Farmed rodents

D (3), HNR-VI (2), S7

(1), CHG5 (1)

D (15), Peru 11 (1), HNR-IV (1), HNR-V(1)

D (18), HNR-VI (2), S7

(1), CHG5 (1), Peru 11

(1), HNR-IV (1),

HNR-V(1)

D (42), PigEbITS7

(19), HNR-VII (15),

Type IV (5), HNR-III

(2), HNR-VI (2); EbpA

(1), Peru 8 (1), Peru 11

(1), ESH-02 (1), S7 (1), CHG5 (1), HNR-I (1),

HNR-II (1), HNR-IV

(1), HNR-V (1) 


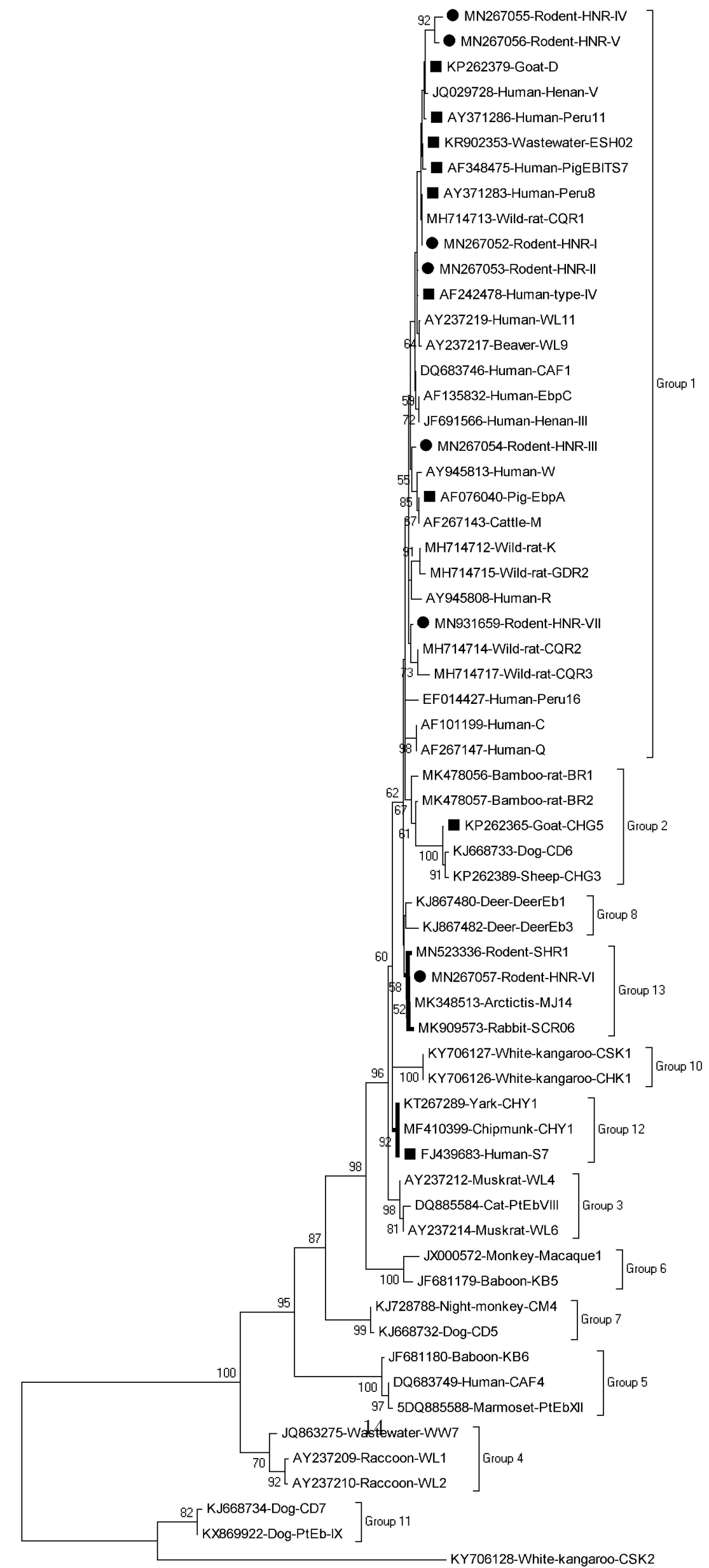

